\title{
Laparoscopic removal of an eroding Mirena coil through the sigmoid colon
}

Hong Kong Med J 2014;20:82

DOI: 10.12809/hkmj134203

To the Editor-I read with interest the recently published article by Hussain et al, ${ }^{1}$ which reported a case of translocated intrauterine device (IUD) removed laparoscopically. I am most curious to know the meaning of "inconclusive X-rays" as stated by the authors. As Mirena is radiopaque, it can either be seen or not seen on X-rays, rather than inconclusive. For the purpose of illustration, I have included an X-ray of a 33-year-old woman who had a partially embedded Mirena in the uterine fundus (Fig). Interestingly, on reading an X-ray for a lost IUD, one really has to look for it to identify its presence. Further, in this reported case, if the X-rays were indeed inconclusive, on what basis did the authors decide that the IUD was expelled, without considering other imagings such as computed tomography $(\mathrm{CT})$ ? If the IUD was translocated close to the bowel, CT could also help to determine the presence and the degree of bowel penetration, ${ }^{2-4}$ so that preoperative counselling and preparation could be provided before proceeding with laparoscopy. The inference of "inconclusive X-rays" should have been pursued further, to avoid the surprise in finding at the first laparoscopy and the need for a second laparoscopy to remove the IUD.

\section{Vincent YT Cheung *, FRCOG, FRCSC}

Department of Obstetrics and Gynaecology, Queen Mary Hospital, The University of Hong Kong, Pokfulam, Hong Kong

* Corresponding author: vytc@hku.hk

\section{References}

1. Hussain A, Omar K, El-Hasani S. Laparoscopic removal of an eroding Mirena coil through the sigmoid colon. Hong

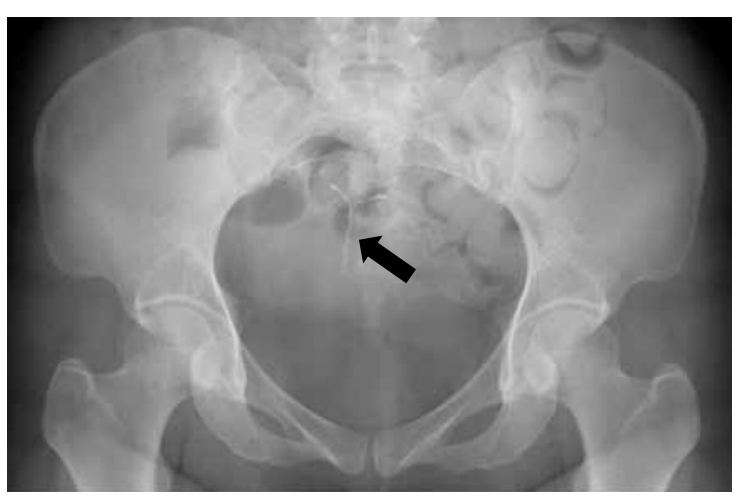

FIG. A pelvic radiograph showing a Mirena intrauterine system (arrow)
Kong Med J 2013;19:560.e3-4.

2. Taras AR, Kaufman JA. Laparoscopic retrieval of intrauterine device perforating the sigmoid colon. JSLS 2010;14:453-5.

3. Zeino MY, Wietfeldt ED, Advani V, Ahad S, Younkin C, Hassan I. Laparoscopic removal of a copper intrauterine device from the sigmoid colon. JSLS 2011;15:568-70.

4. Boortz HE, Margolis DJ, Ragavendra N, Patel MK, Kadell BM. Migration of intrauterine devices: radiologic findings and implications for patient care. Radiographics 2012;32:335-52.

\section{Authors' reply}

To the Editor-Many thanks for Dr Cheung's interest in our article. ${ }^{1}$ First, all intrauterine contraceptive devices are radio-opaque as you correctly point out. The plain abdominal X-ray and ultrasound did not show evidence of the coil according to the reporting radiologists. The reasons were that the coil was unexpectedly located on the pelvic bones (left side) outside the uterine area and the colon was loaded with faeces. Retrospective analysis (after laparoscopy) and re-checking of the plain film actually showed the coil on the left side of the lower abdomen (outside the uterine area). Second, computed tomography (CT) had not been requested for two reasons. According to our hospital protocol, CT is to be avoided in young patients to reduce radiation risks, unless it is highly warranted for an acute abdomen or a lifesaving procedure. The other reason for laparoscopy and not CT was that this patient had lower abdominal pain and vaginal bleeding, of which gynaecological features are commonly investigated laparoscopically due to superior diagnostic yields than any form of imaging (including CT). Hence this woman was in need of a laparoscopy anyway and therefore there was no point of exposing her to the unnecessary risk of radiation via a $\mathrm{CT}$.

A Hussain *,1, FRCS (Eng), FRCS, K Omar ${ }^{2}$, FRCOG, S El-Hasani ${ }^{1}$, FRCS (Eng), FRCS

${ }^{1}$ General Surgery Department

${ }^{2}$ Obstetric and Gynaecology Department

Princess Royal University Hospital, Farnborough Common, Orpington, Kent, BR6 8ND, United Kingdom

* Corresponding author: azahrahussain@yahoo.com

\section{Reference}

1. Hussain A, Omar K, El-Hasani S. Laparoscopic removal of an eroding Mirena coil through the sigmoid colon. Hong Kong Med J 2013;19:560.e3-4. 